\title{
Optimal Identifying Codes in Oriented Paths and Circuits
}

\author{
Ahmed Semri \\ LaROMaD, Faculty of Mathematics, USTHB, \\ BP 32 Bab Ezzouar,El-Alia 16111, \\ Algiers, Algeria.
}

\author{
Hillal Touati \\ LaROMaD, Faculty of Mathematics, USTHB, \\ BP 32 Bab Ezzouar,El-Alia 16111, \\ Algiers, Algeria.
}

Received: February 14, 2021. Revised: March 7, 2021. Accepted: March 10, 2021. Published: March 26, 2021.

\begin{abstract}
Identifying codes in graphs are related to the classical notion of dominating sets [1]. Since there first introduction in 1998 [2], they have been widely studied and extended to several application, such as: detection of faulty processor in multiprocessor systems, locating danger or threats in sensor networks.

Let $\mathbf{G}=(\mathrm{V}, \mathbf{E})$ an unoriented connected graph. The minimum identifying code in graphs is the smallest subset of vertices $C$, such that every vertex in $\mathrm{V}$ have a unique set of neighbors in C. In our work, we focus on finding minimum cardinality of an identifying code in oriented paths and circuits.
\end{abstract}

Keywords: Identifying code, Oriented paths, Circuits.

\section{INTRODUCTION}

After their introduction for modelize the problem of detecting a failures in multiprocessors system[2], the notion of identifying code have been also used in numerious applications such as locating and detecting danger or threats in indoor environments [3] and wireless Network Monitoring [4]. Actually, the theories and the applications of identifying code attracted the attention of many researchers. This led to many results that have been obtained in hypercubes [5], [6], grids [7], [8], paths and cycles [9], [10], [11], [12]. An updated bibliography on the subject can be found in [13].

Let $G=(V, E)$ a simple, connected and undirected graph, where $V$ is the set of vertices and $E$ the set of edges. We call a code any noempty subset of vertices and its elements a codewords. We def ne $B_{r}(v)$, a ball of center $v$ and radius $r$ by $B_{r}(v)=\{u \in V \mid d(u, v) \leq r\}$, where $d(x, y)$ denotes the length (number of edges) of the shortest path between the vertices $x$ and $y$.

Thus, an $r$-identifying code is any subset $C \subseteq V$ such that:

1) $\forall v \in V, B_{r}(v) \cap C \neq \emptyset$,

2) $B_{r}(u) \cap C \neq B_{r}(v) \cap C$, for all $u, v \in V, u \neq v$.

Therefore, the frst condition ensures that every vertice of the graph is covered by at least one codeword, and the second one ensures that every pair of different vertices is separated. In other words, each vertex of the graph $G$ is covered by a unique set of codewords. The set $B_{r}(v) \cap C$, denoted also by $I_{r}(v)$, is called the $r$-identifying set of $v$ (simply identifying set when $r=1$ ).

For an oriented graph $G=(V, A)$, we just replace $B_{r}(v) \cap C$ by $\Gamma_{r}^{-}(v) \cap C=I_{r}^{-}(v)$, where the set $\Gamma_{r}^{-}[x]=\{y \in V \mid$ $d(y, x) \leq r\}$ contains all the predecessors at distance at most $r$ from $x$ ( $x$ within).

The problem with identifying code is f nding one with the fewest elements. This problem is known to be an NP-complete problem [14].

Our work studies this problem in oriented graphs, particularly in oriented paths and circuits. Thus, some partial results were obtained.

\section{Identifying CODE IN ORIENTED PATHS}

As mentioned before, we are interested in fnding an optimal identifying code in oriented paths and circuits. First, we give some notations that will be used in the next paragraphs.

We denote by $\mathcal{P}_{n}$ an oriented path of length $n$, ie it contains exactly $n+1$ vertices, and $\mathcal{C}_{n}$ a circuit of length $n$. Let $M_{r}^{-}(G)$ denotes the minimum cardinality of an $r$-identifying code in graph $G$.

First, we investigate the 1-identifying code (or simply identifying code, if there's no ambiguity) then the 2-identifying code.

\section{A. 1-Identifying Code}

Lemma 1: A subset $C \subseteq V$ is an identifying code in $\mathcal{P}_{n}$ if and only if: 1 .

1) The two vertices $x_{0}$ and $x_{1}$ belong to the code $C$,

2) For every pair of consecutive vertices $x_{i}$ and $x_{i+1}$, $i \in\{2,3, \ldots, n-1\}, x_{i}$ or $x_{i+1}$ is a codeword.

$3)$ For every triplet of consecutive vertices $x_{i}, x_{i+1}$ and $x_{i+2}, i \in\{2,3, \ldots, n-2\}, x_{i}$ or $x_{i+2}$ is a codeword.

Proof: For (1), $x_{0}$ is covered by itself, then $x_{0}$ must be a codeword. In addition, $x_{1}$ must belong to code to separate the pairs of vertices $\left(x_{0}, x_{1}\right)$.

For the second condition, suppose that $x_{i} \notin C$ and $x_{i+1} \notin C$. Then $\mathcal{I}^{-}\left(x_{i+1}\right)=\emptyset\left(x_{i+1}\right.$ isn't covered $)$. Then either $x_{i}$ or $x_{i+1}$ must belong to the code.

For (3), suppose that neither $x_{i}$ nor $x_{i+2}$ belong to the code. Then we have two cases:

Case 1 If $x_{i+1} \in C$, then $\mathcal{I}^{-}\left(x_{i+1}\right)=\mathcal{I}^{-}\left(x_{i+2}\right)=$ $\left\{x_{i+1}\right\}$, ie the two vertices $x_{i+1}$ and $x_{i+2}$ aren't seperated. 
Case 2: If $x_{i+1} \notin C$, necesseraly the two vertices $x_{i+1}$ and $x_{i+2}$ will not be covered because $\mathcal{I}^{-}\left(x_{i+1}\right)=$ $\mathcal{I}^{-}\left(x_{i+2}\right)=\emptyset$.

Thus, in the two cases either $x_{i}$ or $x_{i+2}$ must be a codeword. One can see the necessity and the suff ciency of the three conditions to cover all the vertices of $\mathcal{P}_{n}$, this comes from the fact that every semi-ball contains exactly two consecutive vertices.

Now, let's show the suff ciency of the three conditions for the separation.

Let $x_{i}$ and $x_{j}$ be two vertices, then we have two cases:

Case 1 The vertices are neighbours. Without loss of generality, we put $j=i+1$. Above, we have shown that Condition (1) separates the vertices $x_{0}$ and $x_{1}$. Therefore, by Condition (3), we known that $x_{i-1} \in C$ or $x_{i+1} \in C$, then we have $\mathcal{I}^{-}\left(x_{i}\right) \neq$ $\mathcal{I}^{-}\left(x_{j}\right)$. Thus, $x_{i}$ and $x_{j}$ were separated.

Case $2 x_{i}$ et $x_{j}$ are not neighbours, ie the distance $d\left(x_{i}, x_{j}\right) \geq 2$.

Suppose, without loss of generality, that $j=$ $i+2$. Then, we have $\Gamma_{1}^{-}\left[x_{i}\right]=\left\{x_{i-1}, x_{i}\right\}$ and $\Gamma_{1}^{-}\left[x_{j}\right]=\left\{x_{i+1}, x_{i+2}\right\}$, but by Condition (2), we have $\mathcal{I}^{-}\left(x_{i}\right) \neq \mathcal{I}^{-}\left(x_{j}\right)$. Then $x_{i}$ and $x_{j}$ are separated.

This completes the proof of the lemma.

By the following theorem we give a minimum cardinality of an identifying code in oriented paths.

Theorem 1: For an oriented path $\mathcal{P}_{n}$, we have:

$$
M_{1}^{-}\left(\mathcal{P}_{n}\right)= \begin{cases}2 p & \text { if } \mathrm{n}=3 \mathrm{p}, \\ 2 p+1 & \text { if } \mathrm{n}=3 \mathrm{p}+1, \\ 2 p+2 & \text { if } \mathrm{n}=3 \mathrm{p}+2\end{cases}
$$

Proof: Let $V$ the set vertices of $\mathcal{P}_{n}$. If we denote by $L$ the set of vertices identif ed by one codeword (or covered by one codeword). Then, the other vertices $(|V|-|L|)$ are covered by at least two codewords. In other words, $C$ double covers these vertices. Thus, using the fact that $|L| \leq|C|$ (at most $|C|$ vertices are covered by one codeword), therefore we have the following inequality

$$
2(|V|-|L|)+|L| \leq \sum_{x_{i} \in C}\left|\Gamma_{1}^{-}\left[x_{i}\right]\right| \leq 2 .|C|
$$

so

$$
\begin{aligned}
2|V|-|L| \leq 2|C| & \Leftrightarrow 2|V|-|C| \leq 2|C| \\
& \Leftrightarrow \frac{2}{3}|V| \leq|C|
\end{aligned}
$$

which leads to

$$
|C| \geq\left\lceil\frac{2 n}{3}\right\rceil
$$

Let $n=3 p+q$, with $q \in\{0,1,2\}$. Thus we obtain:

$$
\left\lceil\frac{2 n}{3}\right\rceil=\left\lceil\frac{2(3 p+q)}{3}\right\rceil=2 p+\left\lceil\frac{2 q}{3}\right\rceil
$$

Therefore: If $q=0$, then $\left\lceil\frac{2 q}{3}\right\rceil=0$. If $q=1$, then $\left\lceil\frac{2 q}{3}\right\rceil=1$. And f naly, if $q=2$, then $\left\lceil\frac{2 q}{3}\right\rceil=2$.

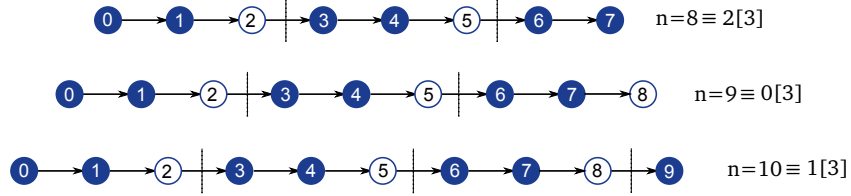

Fig. 1 - An Example of identifying code in oriented paths of length 7,8 and 9

To conclude, we exhibit an identifying code which reaches the bound for each case. Thus, we can choose $C=\left\{x_{i} \mid i \equiv\right.$ $0[3]$ and $i \equiv 1[3]\}$ for all cases (see f gure 1 ).

\section{B. 2-Identifying Code}

Before proceding to the proof of our results we need the following result:

Let $\mathcal{P}_{n}=\left\{x_{0}, x_{1}, \ldots, x_{n}\right\}$ an oriented path of length $n$, and $C$ a code in $\mathcal{P}_{n}$

Lemma 2: A subset $C$ is a 2-identifying code in $\mathcal{P}_{n}$ if and only if the following three conditions are satisf ed: 1 .

1) The vertices $x_{0}, x_{1}$ and $x_{2}$ must belong to $C$,

2) For every group of three consecutive vertices, $x_{i}, x_{i+1}, x_{i+2}, i \in\{3,4, \ldots, n-2\}$, at least one belong to the code $C$,

3) For every group of four consecutive vertices, $x_{i}, x_{i+1}, x_{i+2}, x_{i+3}, i \in\{3,4, \ldots, n-3\}$, we can't have $x_{i} \notin C$ and $x_{i+3} \notin C$.

Proof: For the condition (1), if $x_{0} \notin C$, then we have $\Gamma_{2}^{-}\left[x_{0}\right]=\emptyset$ (the vertex is not covered). Thus $x_{0}$ must be a codeword.

For (2), we can see that if any of the three vertices $x_{i}, x_{i+1}, x_{i+2}$, for all $i \in\{3,4, \ldots, n-2\}$, is not a codeword then $\mathcal{I}^{-}\left(x_{i+2}\right)=\emptyset$ which contradicts the fact that $C$ is a covering code.

Finaly for the condition (3), suppose that neither $x_{i}$ nor $x_{i+3}$ is in $C$ then the two vertices $x_{i+2}$ and $x_{i+3}$ will not be separated because $\mathcal{I}_{2}^{-}\left(x_{i+2}\right)=\mathcal{I}_{2}^{-}\left(x_{i+3}\right)=\left\{x_{i+1}, x_{i+2}\right\}$. Thus $x_{i} \in C$ or $x_{i+3} \in C$, for all $i \in\{3,4, \ldots, n-2\}$.

We remark that the condition (1) and (2) are necessary and suff cient for the condition that $\Gamma_{2}^{-}\left[x_{i}\right] \cap C \neq \emptyset$ for all $i \in\{0,1, \ldots, n\}$.

We need to show that the three conditions of the previous lemma are suff cient for the separation. Let $x_{i}$ and $x_{j}$ be two distinct vertices. Thus, two cases appear:

Case 1 The two vertices are neighbours, $j=i+1$. In this case, by (1) we have the pairs $\left(x_{0}, x_{1}\right),\left(x_{1}, x_{2}\right)$, $\left(x_{2}, x_{3}\right)$ seperated, and by the condition (3), we have $x_{i-3} \in C$ and $x_{i} \in C$ for all pairs $\left(x_{i}, x_{i+1}\right)$, where $i \in\{3,4, \ldots, n-1\}$. We can observe that $\mathcal{I}_{2}^{-}\left(x_{i}\right) \neq \mathcal{I}_{2}^{-}\left(x_{i+1}\right)$ for all pairs of consecutive vertices. Thus, the vertices $x_{i}$ and $x_{j}$ are separated by the code $C$.

Case 2 The two vertices $x_{i}$ and $x_{j}$ are at distance at least 2 , ie $d\left(x_{i}, x_{j}\right) \geq 2$. In this case, if $d\left(x_{i}, x_{j}\right)>2$ $(j>i+2)$, then by (2) we have $\mathcal{I}_{2}^{-}\left(x_{i}\right) \neq$ 
$\mathcal{I}_{2}^{-}\left(x_{j}\right)$, and if $d\left(x_{i}, x_{j}\right)=2$, then by the conditions (2) and (3) we have also $\mathcal{I}_{2}^{-}\left(x_{i}\right) \neq \mathcal{I}_{2}^{-}\left(x_{j}\right)$. Thus, in this case, also $x_{i}$ and $x_{j}$ are separated by the code $C$.

For more clearness, we will denote each vertex $x_{i}$ by its subscript $i$.

We know that each 2-identifying code is 2-separator in $\mathcal{P}_{n}$. Also, we know that for each vertex $i \in \mathcal{P}_{n}(i \geq 2)$ we have $\Gamma_{2}^{-}[i] \Delta \Gamma_{2}^{-}[i+1]=\{i-2, i+1\}^{1}$, then $\forall i \in \mathcal{P}_{n}$ one of the vertices $i-2$ and $i+1$ must belong to $C$ (condition (3) of lemma 2). Thus, we have $i-2 \in C$ or $i+1 \in C$ for each vertex $i \in\{2, n-1\}$ from $\mathcal{P}_{n}$ (see fgure 2). Such disjunction will be called (ie $i-2 \in C$ or $i+1 \in C$ ) Elementary Constraint (EC), so it is abbreviated as $i-2 \vee i+1$.

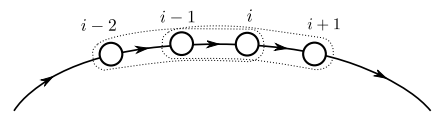

Fig. 2 - One of the two vertices $i$ or $i+3$ belong to a code to separate $i+2$ and $i+3$

Next, we introduce an example which clear up some notations that we will use in the rest of this paper.

Example 1: Let $\mathcal{P}_{10}=x_{0}, x_{1}, \ldots, x_{9}$, an oriented path of length 9 . To obtain a 2 -identifying code we have to separate nine pairs of consecutive vertices. Thus, we have nine ECs to satisfy those we enumerate as follows : $0 \vee 3,3 \vee 6,6 \vee$ $9,1 \vee 4,4 \vee 7,2 \vee 5,5 \vee 8$. Note that we omit the two ECs which separate the pairs $(0,1)$ et $(1,2)$, because they are separated (condition (1) of lemma 2). The set of these constraints will be called General Constraint (GC). This set of elementary constraints (or GC) can partitioned in three subsets of constraints, called Partial Constraints (PC), such as :

$$
\begin{aligned}
& 0 \vee 3,3 \vee 6,6 \vee 9 \\
& 1 \vee 4,4 \vee 7 \\
& 2 \vee 5,5 \vee 8
\end{aligned}
$$

However, in order to get a general formulation, we give some adaptation for this notation. Thus, we can write the above PCs as follows:

$0 \vee 0+1 \times 3,0+1 \times 3 \vee 0+2 \times 3,0+2 \times 3 \vee 0+3 \times 3$

$1 \vee 1+1 \times 3,1+1 \times 3 \vee 1+2 \times 3 \quad\left(C_{1}\right)$

$2 \vee 2+1 \times 3,2+1 \times 3 \vee 2+2 \times 3 \quad\left(C_{2}\right)$

we call $C_{i}, i=0,1,2$, the partial constraint $i$.

In general, if the number of vertices is $n$, then we suppose that $n=3 p+q$, with $p \in\left\{1,2, \ldots,\left[\frac{n}{3}\right]\right\}$ and $q \in\{0,1,2\}$. Thus the PC $i$ has the following form:

$i \vee i+1 \times 3, i+1 \times 3 \vee i+2 \times 3, \ldots, i+\left(s_{i}-1\right) \times 3 \vee i+s_{i} \times 3$

\footnotetext{
${ }^{1} A \Delta B=A \cup B \backslash A \cap B$, called symmetric difference
}

where $s_{i}$ is the greatest integer for which the following inequality checked:

$$
i+s_{i} \times 3 \leq n \text {. }
$$

In the above example, we have $s_{0}=3$ for the PC $C_{0}$, .

Let $V_{i}$ be the set of vertices in the PC $i$. We remark that $V_{i} \cap V_{j}=\emptyset$, for all $i \neq j \mid i, j \in\{0,1,2\}$. In other word, all the PCs have disjoint sets of vertices.

Thus, satisfying the GC, to obtain an 2-identifying code, amounts to satisfy all PCs.

Using this notation, we get the following result:

Theorem 2: Given an oriented path $\mathcal{P}_{n}$ of length $n$, where $n=3 p+q$ and $q \in\{0,1,2\}$. If $C$ is an 2-identifying code in $\mathcal{P}_{n}$. Then:

$$
\begin{aligned}
& \text { If } p=0 \text {, then } M_{2}^{-}\left(\mathcal{P}_{n}\right)=q+1 \\
& \text { If } q=0, p \geq 1, M_{2}^{-}\left(\mathcal{P}_{n}\right)= \\
& \begin{cases}\frac{3 p}{2}+1 & \text { if } p \text { is even } \\
\frac{3(p+1)}{2} & \text { if } p \text { is odd }\end{cases} \\
& \text { If } q=1, p \geq 1, M_{2}^{-}\left(\mathcal{P}_{n}\right)= \\
& \begin{cases}\frac{3 p}{2}+2 & \text { if } p \text { is even } \\
\frac{3(p+1)}{2} & \text { otherwise }\end{cases} \\
& \text { If } q=2, p \geq 1, M_{2}^{-}\left(\mathcal{P}_{n}\right)= \\
& \begin{cases}\frac{3 p}{2}+2 & \text { if } p \text { is even } \\
\frac{3(p+1)}{2}+1 & \text { otherwise }\end{cases}
\end{aligned}
$$

Proof: $\quad$ If $p=0(n \leq 2)$ the minimum cardinality of a 2 -identifying code in $\mathcal{P}_{n}$ is deduced from the frst condition of lemma 2.

For the second case, ie $p \geq 1$ and $q=0$, we know, by the condition (1) of lemma 2, that the vertices 0,1 and 2 belong to the code, which satisf es the frst EC of the PCs 0,1 and 2. On the other hand, we have, by condition (2) of lemma 2, necessary at least one codeword between 3,4 and 5 , thus we have one EC between the PCs 0,1 and 2 for which two vertices are a codeword. Without loss of generality, let 3 this vertex, then this satisf es two ECs in the PC 0. In this case, we need to satisfy $(p-3) \mathrm{ECs}$, then at least $\left\lceil\frac{p-3}{2}\right\rceil$ codeword are needed to satisfy the rest of ECs in PC 0 .

In addition, for each of the partial constraints 1 and 2 we have one elementary constraint satisf ed (since $1,2 \in C$ ), then $(p-2)$ ECs aren't satisf ed for each one. Thus, at least $\left\lceil\frac{p-2}{2}\right\rceil$ codewords are needed to satify the rest of ECs for PCs 1 and 2 .

$\left(C_{0}\right)$ We conclude that we need, totally, at least:

$$
4+2\left\lceil\frac{p-2}{2}\right\rceil+\left\lceil\frac{p-3}{2}\right\rceil
$$

codewords to satisfy the general constraint.

If $p$ is even, then $M_{2}^{-}\left(\mathcal{P}_{n}\right) \geq \frac{3 p}{2}+1$. Else, $M_{2}^{-}\left(\mathcal{P}_{n}\right) \geq$ $\frac{3(p+1)}{2}$.

Finally, we construct a 2-identifying code that reaches the bound to conclude. Indeed, we use the following construction: We take all vertices $i \in V$, where $i$ is even and adding vertices 0 and 1.

The proof of the case $p \geq 1$ and $q=1$ is similar. Indeed, concerning the frst EC of CP 0 , we have $(p-1)$ ECs to satisfy, 


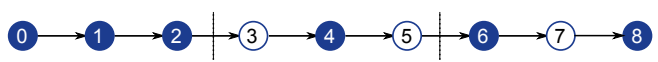

Fig. 3 - 2-identifying code $(C=\{2,4,6,8\} \cup\{0,1\})$ for an oriented path of length 8 ( $n=3 \times 3+0$ vertices $)$

since $0 \in C$. Thus, we need at least $\left\lceil\frac{p-1}{2}\right\rceil$ codewords. For the PCs 1 and 2 we need, respectively, at least $\left\lceil\frac{p-3}{2}\right\rceil$ and $\left\lceil\frac{p-2}{2}\right\rceil$ codewords to satisfy the rest of elementary constraints. Thus, we need at least :

$$
4+\left\lceil\frac{p-3}{2}\right\rceil+\left\lceil\frac{p-2}{2}\right\rceil+\left\lceil\frac{p-1}{2}\right\rceil
$$

codeword.

Then, if $p$ is even, then $M_{2}^{-}\left(\mathcal{P}_{n}\right) \geq \frac{3 p}{2}+2$. Else, $M_{2}^{-}\left(\mathcal{P}_{n}\right) \geq \frac{3(p+1)}{2}$.

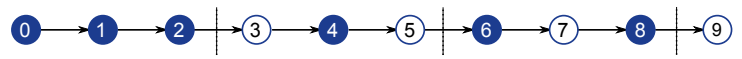

Fig. 4 - A 2-identifying in an oriented path having $n=3 \times$ $3+1$ vertices $(p=3, q=1)$

To conclude, we exhibit a code reaching these bound. Indeed, we remark that for the code $C=\{i \mid i$ is even $\} \cup\{0,1\}$ the bound is attained (see fgure 4).

Finally, the proof for the last case $(p \geq 1$ and $q=2)$ is also similar, we have $(p-1)$ ECs to satisfy for the PCs 0 and 1 , and we need respectivey at least $\left\lceil\frac{p-1}{2}\right\rceil$ and $\left\lceil\frac{p-2}{2}\right\rceil$ codewords. For the PC 2 we have $(p-1) \mathrm{EC}$, since $2 \in C$ then we have $(p-2)$ ECs to satisfy, then at least $\left\lceil\frac{p-2}{2}\right\rceil$ codewords are needed. Thus, we need at least :

$$
4+2\left\lceil\frac{p-2}{2}\right\rceil+\left\lceil\frac{p-1}{2}\right\rceil
$$

codewords to satisfy the GC.

If $p$ is even, then $M_{2}^{-}\left(\mathcal{P}_{n}\right) \geq \frac{3 p}{2}+2$. If $p$ is odd, then $M_{2}^{-}\left(\mathcal{P}_{n}\right) \geq \frac{3(p+1)}{2}$. To conclude, we just consider the same construction as the previous cases to exhibit a 2-identifying code reaching the bound.

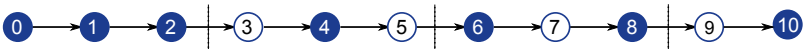

Fig. 5 - An Example of 2-identifying code in an oriented path with $3 \times 3+2=11$ vertices $(p=3, q=2)$

\section{IDENTIFYING CODE IN CIRCUITS}

In circuits we give an optimal 2-identifying code.

\section{A. 2-Identifying Code}

In the case of circuit, the two conditions of the lemma 2 are still valid. Then:
Lemma 3: Let $\mathcal{C}_{n}=\{1,2, \ldots, n, 1\}$ a circuit of length $n$. $C$ is a 2 -identifying code for $\mathcal{C}_{n}$ if and only if the following conditions are satisf ed:

1) For all group of three consecutive vertices $x_{i}, x_{i+1}$ and $x_{i+2}$ at least one of them is a codeword,

2) For all group of four consecutive vertices $x_{i}, \ldots, x_{i+3}$ we could not have $x_{i} \notin C$ and $x_{i+3} \notin C$.

Proof: The proof is similar to lemma 2. Except adding the condition that the distance between $i$ and $j$ isn't greater than that between $j$ and $i$ to show the suff ciency and the necessity of the conditions (1) and (2).

In the case of circuit, Although the reasoning is similar as in oriented path, there is, however, some differences. Thus, we def ne a partial constraint $i(i \in\{1,2,3\})$ as follow:

$i \bigvee i+1 \times 3, i+1 \times 3 \bigvee i+2 \times 3, \ldots, i+\left(s_{i}-1\right) \times 3 \bigvee i+s_{i} \times 3, i+s_{i} \times 3 \bigvee h_{i}$ where $s_{i}$ is the greatest integer such:

$$
i+s_{i} \times 3 \leq n
$$

and $h_{i}$ is such that $i+\left(s_{i}+1\right) \times 3 \equiv h_{i}(\bmod [n])$, (ie $\left.h_{i} \in\{1,2,3\}\right)$.

Example 2: Let $\mathcal{C}_{n}=\{1,2, \ldots, n, 1\}$ be a circuit of length $n$. Suppose that $n=10$, thus $p=3$ and $q=1$. Then the PCs, 1,2 et 3 , can be written as follows:

$$
\begin{aligned}
& 1 \vee 4,4 \vee 7,7 \vee 10,10 \vee 3, \quad(i=1) \\
& 2 \vee 5,5 \vee 8,8 \vee 1 \quad(i=2) \\
& 3 \vee 6,6 \vee 9,9 \vee 2 \quad(i=3)
\end{aligned}
$$

denoting by $i \mid j$ the elementary constraint $i \vee j$. Then the GC is written:

$$
1|4| 7|10| 3|6| 9|2| 5|8| 1
$$

If, for example, $n=12$, then the $\mathrm{GC}$ will be:

$$
1|4| 7|10| 1,2|5| 8|11| 2,3|6| 9|12| 3
$$

Thus, we have determined the optimal 2-identifying code. The result is given by the following theorem:

Theorem 3: Let $C_{n}$ be a circuit of length $n$. Then:

$$
M_{2}^{-}\left(C_{n}\right)= \begin{cases}\emptyset & \text { if } n \leq 3, \\ 3 & \text { if } n=4, \\ k & \text { if } n=2 k, k \geq 3, \\ k+1 & \text { if } n=2 k+1, k \geq 2,\end{cases}
$$

Proof: For (1), it is clear that, if $n \leq 3$, then $\mathcal{C}_{n}$ can't admit a 2 -identifying code because there are twin vertices ${ }^{2}$.

\footnotetext{
${ }^{2}$ We call twin vertices every two vertices $u, v$ such that $\Gamma_{r}^{-}(u)=\Gamma_{r}^{-}(v)$
} 
For (2), we show that there is no 2-identifying code of cardinality 2 in a circuit of length 4 . Indeed, suppose that there are only two vertices as codeword. Without loss of generality, let 1 and 3 be these vertices, then $I_{2}^{-}(1)=I_{2}^{-}(3)=\{1,3\}$. Therefore at least three vertices must belong to a code. Finally, it suff ces to exhibit a code with cardinality 3 to conclude (see f gure 6).

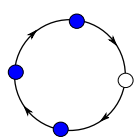

Fig. 6 - Exemple de code 2-identifiant dans $\mathcal{C}_{4}$

Concerning (3), ie the case where the length of the circuit is even $(n=2 k)$, we know that there is $\frac{n}{2}=k$ ECs to satisfy, therefore we need at least $k$ codewords.

It suff ces to exhibit a 2-identifying code of cardinality $k$ to conclude. Thus, we can take as a code the set $C=$ $\{i \mid i$ even, $1 \leq i \leq n\}$ (see the fgure 7) hence the result.

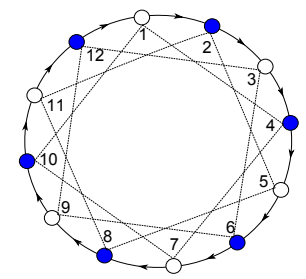

Fig. 7 - An example of 2-identifying code in circuit of length 12. Six codewords are needed to cover and separate all the vertices of the circuit.

Similarly to the previous case, when $n=2 k+1$ (n is odd) we need at least $k+1$ codewords to satisfy all the ECs.

Thus, there is at least one EC which has its vertices belong to the code. We want to show that $|C|>k+1$. To do it, we suppose that we can $\mathrm{f}$ nd a 2 -identifying code $C$ of cardinality $k+1$ in a circuit of length $n$, and we get to a contradiction. Since we have $k+1$ vertices as codewords, then necessarily two codewords are adjacent. Without loss of generality, let 1 and 2 these two vertices, or one of the two vertices $n$ and 3 must be a codeword by the condition (2) of lemma 3. Thus, for every 2 -identifying code at least three consecutive vertices are codewords.

Now, there are two cases:

Case 1: Suppose that the length of the circuit is equal to $n=4 p+3(k=2 p+1)$. Since at least three consecutive vertices are codewords ( as mentioned previously), then we need to cover and separate $4 p$ vertices. But by conditions (1) and (2) of lemma 3, we know that for every four consecutive vertices, at least two of them are codewords. Thus, we need at least $2 p$ vertices as codeword, therefore $2 p+3=k+2$ vertices belong to a code (see the example of the f gure 8 ). Hence the contradiction.

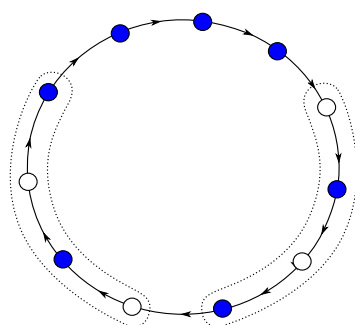

Fig. 8 - An example of optimal 2-identifying code of cardinality $2 \times 2+3$ in circuit of length $4 \times 2+3$

Case 2: In this case, we have $n=4 p+1(k=2 p)$. Observing this case, we see that it's similar to the frst one. We have $n=4 p+1=[4(p-1)+$ $3]+2(k=2 p)$. Thus, $2(p-1)+3=k+1$ vertices are codewords among the $4(p-1)+3$ vertices that a circuit contains (by condition (2) of lemma 3 ) adding the three consecutive vertices belong to the code.

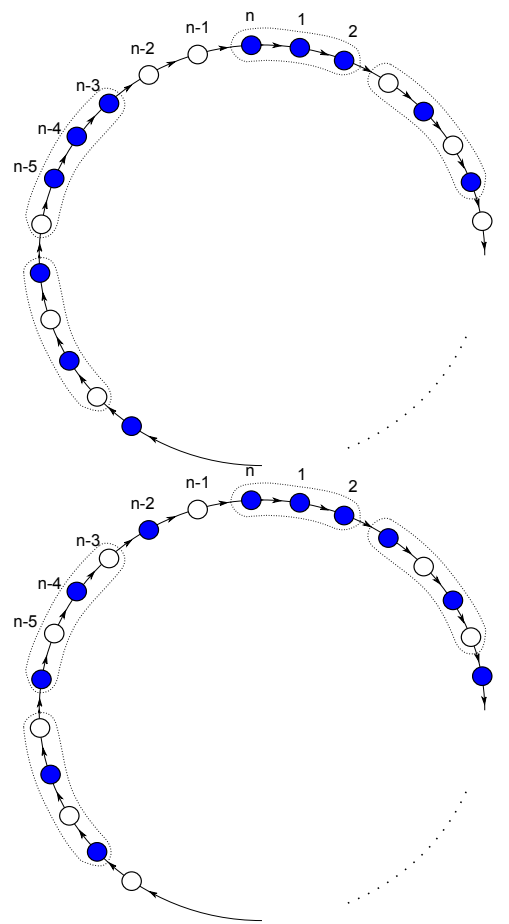

Fig. 9 - An example of two optimal 2-identifying code

In the case where the remaining two vertices don't belong to the code, saying, without loss of generality, $n-1$ and $n-2$, then necessarely the three vertices $n-3, n-4$ and $n-5$ belong to the code (see the left representation in f gure 9). Thereby, we have $4(p-2)$ vertices that are couvered and separated by $2(p-2)$ codewords plus $n-3, n-4$ et $n-5$ and the three consecutive vertices. In total there is $2(p-2)+6=2 p+2=k+2$ codewords. If, either $n-1$ or $n-2$ belong to the code then, we will need $2(p-1)$ codewords for covering and separating the $4(p-1)$ vertices (conditions of lemma 3 ) adding the three consecutive vertices 
(see the left representation in f gure 9). Thus, in total, we will have $1+2(p-1)+3=2 p+2=k+2$ codewords.

Therefore, in the two cases, we will have at least $k+2$ codewords.

We conclude by exhibiting a 2-identifying code reaching this bound. The latter, constituted of the set of vertices $C=$ $\{2\} \cup\{i \equiv 1[2], 1 \leq i \leq n\}$.

\section{CONCLUSION}

In this work we gave some results about identifying code in oriented paths and circuits. It remains to determine the minimum cardinality for the case of a 1-identifying code in circuit. In addition, the question of the general case, ie $r$ identifying code is also an open problem.

\section{ACKNOWLEDGEMENTS}

The authors wish to thank anonymous reviewers for careful reading and comments which improved the quality of the paper.

\section{REFERENCES}

[1] T. Berger-Wolf, M. Laifenfeld, and A. Trachtenberg, "Identifying Codes and the Set Cover Problem," Annual Allerton Conf. on Comm, Ctrl and Comput $44^{\text {th }}, 2006$.

[2] L. B. Levitin, M. G. Karpovsky, and K. Chakrabarty, "On New Class of Codes for Identifying Vertices in Graphs," IEEE Transactions On Information Theory, vol. 44, no. 2, pp. 599-611, 1998.

[3] S. Ray, D. Starobinski, A. Trachtenberg, and R. Ungrangsi, "Robust Location Detection With Sensor Networks," IEEE Journal on selected areas in communications, vol. 22, no. 6, Aug. 2004.

[4] M. Laifenfeld, A. Trachtenberg, R. Cohen, and D. Starobinski, "Joint Monitoring and Routing in Wireless Sensor Networks Using Robust Identifying Codes," Mobile Networks and Applications, vol. 14, no. 42, pp. 415-432, Aug. 2009.

[5] S. Litsyn, U. Blass, and L. Honkala, "Bounds on Identifying Codes," Discret Mathematics, vol. 241, pp. 119-128, 2001.

[6] V. junnila, G. Exoo, T. Laihonen, and S. Ranto, "Upper Bounds For Binary Identifying Codes," Advances in Applied Mathematics, no. 42, pp. 277-289, 2009

[7] J. Moncel, M. Daniel, and S. Gravier, "Identifying code in some subgraphs of the square lattice," Theoretical Computer Science, vol. 319, pp. 411-421, 2004.

[8] C. Payan, G. Cohen, L. Honkala, S. Gravier, A. Lobstein, and M. Mollard, "Improved Identifying Codes For the Grid," Electronic Journal of Combinatorics, 1999

[9] A. Lobstein, N. Bertrand, I. Charon, and O. Hudry, "Identifying and locating-dominating codes on chains and cycles," European Journal of Combinatorics, vol. 25, pp. 969-987, 2004.

[10] A. Semri, S. Gravier, and J. Moncel, "Identifying codes of cycles," European Journal of Combinatorics, vol. 27, pp. 767-776, 2006

[11] F. S. Roberts and D. L. Roberts, "Locating sensors in paths and cycles: The case of 2-identifying codes," European Journal of Combinatorics, vol. 29, pp. 72-82, 2008.

[12] V. Junnila and T. Laihonen, "Optimal Identifying Codes in Cyclces and Paths," Graphs and Combinatorics, vol. 28, no. 42, pp. 469-481, Jul 2012.

[13] A. Lobstein, http://perso.telecom-paristech.fr/ lobstein/debutBIBidetlocdom.pdf.

[14] A. Lobstein, I. Charon, and O. Hudry, "Minimizing the size of an identifying or locating-dominating code in graph is NP-Hard," Theoretical Computer Science, vol. 290, pp. 2109-2120, 2003.

\section{Creative Commons Attribution License 4.0 (Attribution 4.0 International, CC BY 4.0)}

This article is published under the terms of the Creative Commons Attribution License 4.0

https://creativecommons.org/licenses/by/4.0/deed.en_US 